\title{
FERROFLUID LUBRICATION OF A SLIDER BEARING WITH A CIRCULAR CONVEX PAD
}

RAJESH C.SHAH ${ }^{* 1}$ and M.V.BHAT ${ }^{2}$

1 Department of Mathematics, Nirma Institute of Technology, Sarkhej-Gandhinagar Highway, Ahmedabad - 382 481, Gujarat State, India.

2 E-202, Riddhi Complex, Near Jodhpur Village, Ahmedabad - 380 015, Gujarat State, India.

(Received: 06 August 2002; accepted: 04 December 2003)

\begin{abstract}
Analysis was done of a slider bearing with its stator having a circular convex pad surface, using a ferrofluid lubricant with a Jenkins model to describe its flow. Expressions were obtained for dimensionless pressure, load capacity, friction on the slider, the coefficient of friction and the position of the centre of pressure. The pressure was little affected by either the crown height or the material parameter. However, it increased considerably with increasing values of the field strength. The load capacity increased with increasing values of the field or film thickness ratio and decreasing values of the material parameter. The friction force on the slider decreased when the film thickness ratio increased. However, it increased after a certain value of the film thickness ratio when either the field strength or the material parameter increased. The coefficient of friction increased with increasing values of the material parameter or decreasing values of the film thickness ratio or the field strength. The position of the centre of pressure shifted towards the outlet when the film thickness ratio increased. It shifted towards the inlet when either the field strength or the material parameter increased only after the film thickness ratio attained a certain value.
\end{abstract}

Keywords: Convex pad, ferrofluid lubrication, Jenkins model, slider bearing.

\section{INTRODUCTION}

A bearing with an impermeable stator and an impermeable slider with a convex pad surface was studied by Abramovitz ${ }^{1}$. He found its performance better than that of a plane slider. Vinay Puri and $\mathrm{Patel}^{2}$ generalized the above analysis by taking the stator to have a porous facing of uniform thickness backed by a solid wall. They found that such a bearing had more load capacity, friction and coefficient of friction than the corresponding bearing with a plane slider.

A ferrofluid is a suspension of solid magnetic particles of subdomain size in a liquid carrier. Agrawal ${ }^{3}$, Paras Ram and Verma ${ }^{4}$ studied inclined porous slider bearing with a ferrofluid lubricant using Neuringer- Rosensweig model and Jenkins model respectively to describe the flow. They found that magnetization increased the load capacity of the bearing without altering the friction on the slider. Jenkins considered material property also, thus generalizing the Neuringer-Rosensweig model. Recently, Shah and Bhat ${ }^{5,6}$ considered the effect of magnetic fluid lubricant

\footnotetext{
* Corresponding author
} 
on the squeeze film between curved porous rotating circular plates and two curved annular plates and found that ferrofluid lubricant was more advantageous than the conventional lubricant.

In the present paper the convex slider bearing with a ferrofluid lubricant whose flow is described by Jenkins was studied.

\section{Formulation of the Problem}

The impermeable bearing consists of a stator with a circular convex pad surface with crown height $\delta$ and a slilder moving with a uniform velocity $U$ in the $\mathrm{x}$-direction.

The film thickness $\mathrm{h}$ is taken as

$h=4 \delta\left(\frac{x^{2}}{B^{2}}-\frac{x}{B}\right)+h_{2}-\left(h_{2}-h_{1}\right) \frac{x}{B}$,

where $B$ is the bearing breadth, $h_{1}$ and $h_{2}$ are the minimum and maximum film thicknesses.

Assuming steady flow of the lubricant with no slip condition at the boundaries, no end effects and no side effects, the equation governing the film pressure $\mathrm{p}$ is deduced form Ram and Verma ${ }^{4}$ as

$\frac{d}{d x}\left[\frac{h^{3}}{\left(1-\frac{\rho \alpha^{2} \bar{\mu}}{2 \zeta}\right)} \frac{d}{d x}\left(p-\frac{1}{2} \mu_{0} \bar{\mu} H^{2}\right)\right]=6 \zeta U \frac{d h}{d x}$,

where $\rho$ is the fluid density, $\zeta$ is the fluid viscosity, $\alpha^{2}$ is the material constant of Jenkins model, $\bar{\mu}$ is the magnetic susceptibility of the fluid particles, $H$ is the magnitude of the external magnetic field $\bar{H}$ and $\mu_{0}$ is the free space permeability. If $\tau$ is the stress and $u$ is the fluid velocity in the $x$-direction, the shear-stress relation is

$\tau=\zeta \frac{\partial \mathrm{u}}{\partial \mathrm{z}}$

We take a magnetic field $\overline{\mathrm{H}}$ of strength $\mathrm{H}$ inclined at an angle $\phi$ with the $\mathrm{x}$-axis. It vanishes at the inlet and outlet of the bearing so that it attains its maximum at the middle of the bearing as $\mathrm{p}$ does, thus enhancing the latter. The inclination $\phi$ does not appear in eq. (2) and can be obtained as in Ram and Verma ${ }^{4}$. Thus, we define 
$\mathrm{H}^{2}=\mathrm{K} \mathbf{x}(\mathrm{B}-\mathbf{x})$

$\mathrm{K}$ being a quantity chosen to suit the dimensions of both sides and the value of $H$. Let us introduce the dimensionless quantities

$\overline{\mathrm{x}}=\frac{\mathrm{x}}{\mathrm{B}}, \overline{\mathrm{h}}=\frac{\mathrm{h}}{\mathrm{h}_{1}}, \overline{\mathrm{p}}=\frac{\mathrm{h}_{1}^{2} \mathrm{p}}{\zeta U B}, \mu^{*}=\frac{\mu_{0} \bar{\mu} \mathrm{KBh}_{1}^{2}}{\zeta U}$,

$\bar{\delta}=\frac{\delta}{h_{1}}, a=\frac{h_{2}}{h_{1}}, \beta^{2}=\frac{\rho \alpha^{2} \bar{\mu} \sqrt{K B}}{2 \zeta}$.

Using eqs.(4), (5), eqns. (1) and (2) yield

$\frac{d}{d \bar{x}}\left[G \frac{d}{d \bar{x}}\left\{\bar{p}-\frac{1}{2} \mu^{*} \bar{x}(1-\bar{x})\right\}\right]=6 \frac{d \bar{h}}{d \bar{x}}$,

where

$\overline{\mathrm{h}}=4 \bar{\delta}\left(\overline{\mathrm{x}}^{2}-\overline{\mathrm{x}}\right)+\mathrm{a}-(\mathrm{a}-1) \overline{\mathrm{x}}$

$G=\frac{\bar{h}^{3}}{\left(1-\beta^{2} \sqrt{\bar{x}(1-\bar{x})}\right)}$

\section{Solution}

Solving eq.(6) under the boundary conditions

$\overline{\mathrm{p}}=0$ when $\overline{\mathrm{x}}=0,1$,

we obtain

$\overline{\mathrm{p}}=\frac{1}{2} \mu^{*} \overline{\mathrm{x}}(1-\overline{\mathrm{x}})+\int_{1}^{\overline{\mathrm{x}}} \frac{6 \overline{\mathrm{h}}-\mathrm{Q}}{\mathrm{G}} \mathrm{d} \overline{\mathrm{x}}$

where $Q=\frac{6 \int_{0}^{1} \frac{\bar{h}}{G} d \bar{x}}{\int_{0}^{1} \frac{1}{G} d \bar{x}}$.

The load capacity $W$, friction on the slider $F$, coefficient of friction $f$ and the position of centre of pressure $\mathrm{X}$ are defined as

$$
\mathrm{W}=\int_{0}^{\mathrm{L}} \int_{0}^{\mathrm{B}} \mathrm{p}(\mathrm{x}) \mathrm{dxdy}, \mathrm{F}=\left.\int_{0}^{\mathrm{L}} \int_{0}^{\mathrm{B}} \zeta \frac{\partial \mathrm{u}}{\partial \mathrm{z}}\right|_{\mathrm{z}=0} \mathrm{dxdy}, \mathrm{f}=\frac{\mathrm{F}}{\mathrm{W}}, \mathrm{X}=\frac{1}{\mathrm{~W}} \int_{0}^{\mathrm{L}} \int_{0}^{\mathrm{B}} \mathrm{p} x \mathrm{dxdy},
$$


where $\mathrm{L}$ is the bearing length.

They are expressed in non-dimensional forms as

$$
\begin{aligned}
& \bar{W}=\frac{h_{1}^{2} W}{\zeta U B^{2} L}=\frac{\mu^{*}}{12}-\int_{0}^{1} \bar{x} \frac{6 \bar{h}-Q}{G} d \bar{x}, \\
& \bar{F}=\frac{h_{1} F}{\zeta U B L}=\int_{0}^{1} \frac{8 \bar{h}-Q}{2 \bar{h}^{2}} d \bar{x}, \\
& \bar{f}=\frac{B f}{h_{1}}=\frac{\bar{F}}{\bar{W}}, \\
& \bar{X}=\frac{X}{B}=\frac{1}{\bar{W}}\left[\frac{\mu^{*}}{24}-\frac{1}{2} \int_{0}^{1} \bar{x}^{2} \frac{6 \bar{h}-Q}{G} d \bar{x}\right] .
\end{aligned}
$$

\section{RESULTS AND DISCUSSION}

Expressions for dimensionless pressure $\overline{\mathrm{p}}$, load capacity $\overline{\mathrm{W}}$, friction on the slider $\overline{\mathrm{F}}$, the coefficient of friction $\overline{\mathrm{f}}$ and for the position $\overline{\mathrm{X}}$ of the centre of pressure are given by eqs. (10), (12) - (15).

In the references ${ }^{3-4}$, both the magnetization parameter and material parameter include $\mathrm{K}$, the field strength. As per the referees suggestion to make the material parameter independent of $\mathrm{K}$, we define it by the equation

$$
\bar{\beta}=\frac{\beta^{2}}{\sqrt{\mu^{*}}},
$$

so that $\bar{\beta}$ is independent of $\mathrm{K}$. Then

$G=\frac{\bar{h}^{3}}{1-\bar{\beta} \sqrt{\mu^{*} \bar{x}(1-\bar{x})}}$.

Let us take the representative values of $\mathrm{Bhat}^{7}$ as,

$\mathrm{B}=0.15 \mathrm{~m}, \mathrm{~h}_{1}=0.025 \mathrm{~m}, \mathrm{U}=1 \mathrm{~ms}^{-1}, \mu_{0}=4 \pi \times 10^{-7} \mathrm{kgms}^{-2} \mathrm{~A}^{-2}, \bar{\mu}=0.05$, $\zeta=0.002 \mathrm{kgm}^{-1} \mathrm{~s}^{-1}, \rho=800 \mathrm{~kg} \mathrm{~m}^{-3}$,

to compute the values of the bearing characteristics $\bar{p}, \overline{\mathrm{W}}, \overline{\mathrm{F}}, \overline{\mathrm{f}}$ and $\overline{\mathrm{X}}$ which are displayed in tabular form and graphical forms in Tables 1-7 and Figs. 2-5.

The values corresponding to $K=0$ and $\bar{\beta}=0$ in the above represent the results for a conventional lubricant obtained by Abramovitz ${ }^{1}$ and those for a ferrofluid lubricant flowing following the Neuringer-Rosensweig model respectively. 
From Table 1 we see that $\overline{\mathrm{p}}$ is symmetrical about the line $\overline{\mathrm{x}}=0.5$ and attains a maximum there. Moreover, $\overline{\mathrm{p}}$ is not much affected by the crown height $\delta$.

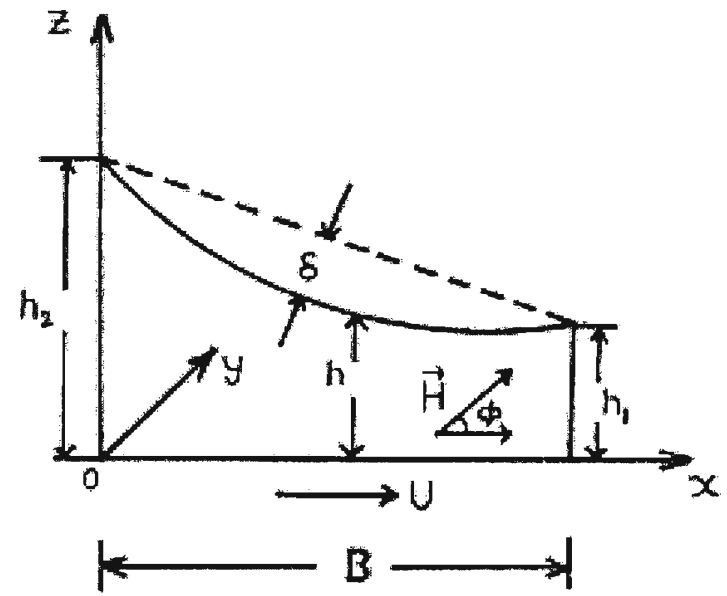

Figure 1: Slider Bearing with a Circular Convex Pad Surface

Table 1: $\bar{p}$ vs $\bar{\delta}$ for various values of $\bar{x}$

\begin{tabular}{|c|c|c|c|c|c|c|}
\hline$\overline{\bar{\delta}}$ & $\overline{\mathrm{x}}$ & 0.1 & 0.3 & 0.5 & 0.7 & 0.9 \\
\hline 0.35 & & 0.1325880 & 0.3093721 & 0.3682999 & 0.3093721 & 0.1325881 \\
\hline 0.15 & & 0.1325880 & 0.3093719 & 0.3682999 & 0.3093718 & 0.1325884 \\
\hline 0.0 & & 0.1325880 & 0.3093719 & 0.3683000 & 0.3093721 & 0.1325879 \\
\hline
\end{tabular}

$\mathrm{a}=2.0, \mathrm{~K}=10^{9}, \bar{\beta}=2.77 \times 10^{-4}$

From Table $2 \overline{\mathrm{p}}$ is not much affected by the material parameter $\bar{\beta}$.

Table 2: $\bar{p}$ vs $\bar{\beta}$ for various values of $\bar{x}$

\begin{tabular}{|c|c|c|c|c|c|c|}
\hline$\alpha^{2}$ & $\overline{\mathrm{x}}$ & 0.1 & 0.3 & 0.5 & 0.7 & 0.9 \\
\hline & & & 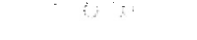 & & & \\
\hline 0.0 & & 0.1325880 & 0.3093719 & 0.3683000 & 0.3093719 & 0.1325880 \\
\hline $10^{-11}$ & & 0.1325880 & 0.3093719 & 0.3682999 & 0.3093718 & 0.1325884 \\
\hline $10^{-10}$ & & 0.1325880 & 0.3093720 & 0.3683000 & 0.3093720 & 0.1325881 \\
\hline $10^{-9}$ & & 0.1325880 & 0.3093720 & 0.3683001 & 0.3093720 & 0.1325881 \\
\hline
\end{tabular}

$\bar{\delta}=0.15, \mathrm{a}=2.0, \mathrm{~K}=10^{9}, \bar{\beta}=2.77 \times 10^{7} \alpha^{2}$ 
From Fig. $2 \overline{\mathrm{p}}$ increases considerably for increasing values of the field strength K. For a ten-fold increase in $\mathrm{K}$, there is a ten-fold increase in $\overline{\mathrm{p}}$.

From Fig. $3 \overline{\mathrm{W}}$ increases for increasing values of $\mathrm{K}$ or a. It can be considerably increased by increasing $\mathrm{K}$.

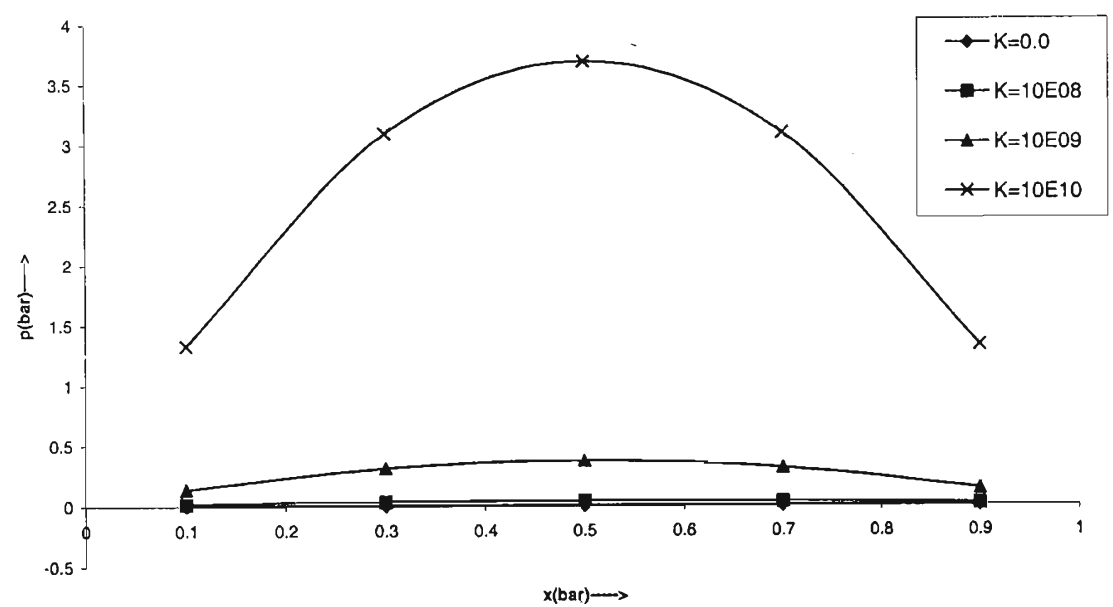

Figure 2: $\bar{p}$ vs $\mathrm{K}$ for various values of $\overline{\mathbf{x}}$ for $\mathrm{a}=\mathbf{2 . 0}, \bar{\delta}=0.15, \bar{\beta}=\mathbf{2 . 7 7 \times 1 0 ^ { - 4 }}$

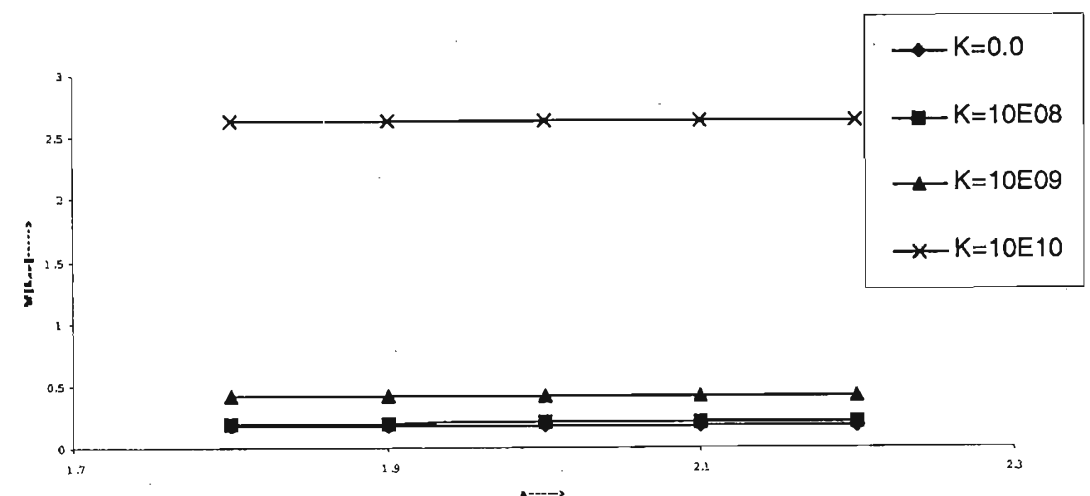

Figure3: $\overline{\mathrm{W}}$ vs K for various values of a for $\bar{\delta}=0.35, \bar{\beta}=2.77 \times 10^{-4}$

From Table $3 \overline{\mathrm{F}}$ decreases when a increases. However, it increases when $\mathrm{K}$ increases provided a $>\mathrm{a}_{0}, 1.8<\mathrm{a}_{0}<1.9$. 
Table 3: $\overline{\mathbf{F}}$ vs $\mathrm{K}$ for various values of a

\begin{tabular}{|c|c|c|c|c|c|c|}
\hline $\mathrm{K}$ & $\mathbf{a}$ & 1.8 & 1.9 & 2.0 & 2.1 & 2.2 \\
\hline 0.0 & & 0.9651934 & 0.9397183 & 0.9170077 & 0.8966164 & 0.8781847 \\
\hline $10^{8}$ & & 0.9651924 & 0.9397184 & 0.9170086 & 0.8966175 & 0.8781862 \\
\hline $10^{9}$ & & 0.9651908 & 0.9397179 & 0.9170097 & 0.8966198 & 0.8781895 \\
\hline $10^{10}$ & & 0.9651859 & 0.9397173 & 0.9170128 & 0.8966268 & 0.8782002 \\
\hline
\end{tabular}

$\bar{\delta}=0.35, \bar{\beta}=2.77 \times 10^{-4}$

From Fig. $4 \overline{\mathrm{f}}$ decreases with increasing values of a or $\mathrm{K}$.

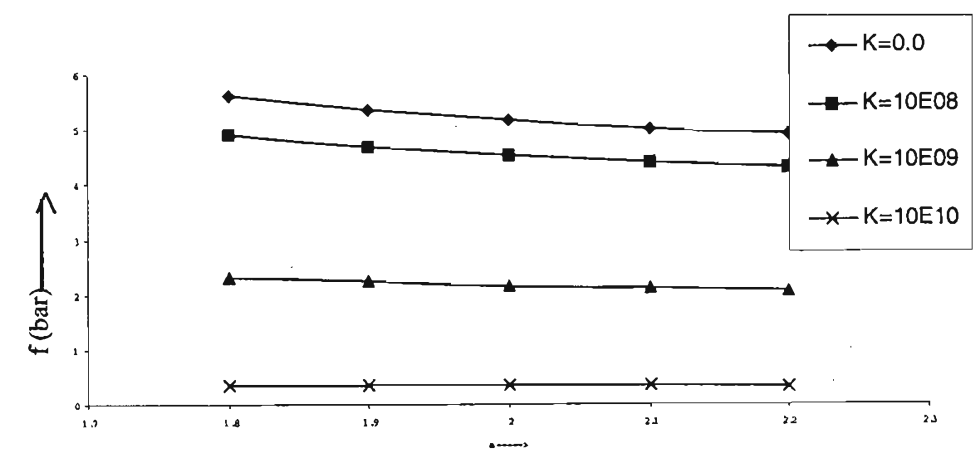

Figure 4: $\overline{\mathrm{f}} \mathrm{vs} \mathrm{K}$ for various values of $\mathrm{a}$ for $\bar{\delta}=0.35, \bar{\beta}=2.77 \times 10^{-4}$

From Fig. 5 the position of the centre of pressure shifts towards the outlet when $\mathrm{K}$ increases. But shifts towards the inlet when $\mathrm{a}>\mathrm{a}_{1}, 1.8<\mathrm{a}_{1}<1.9$.

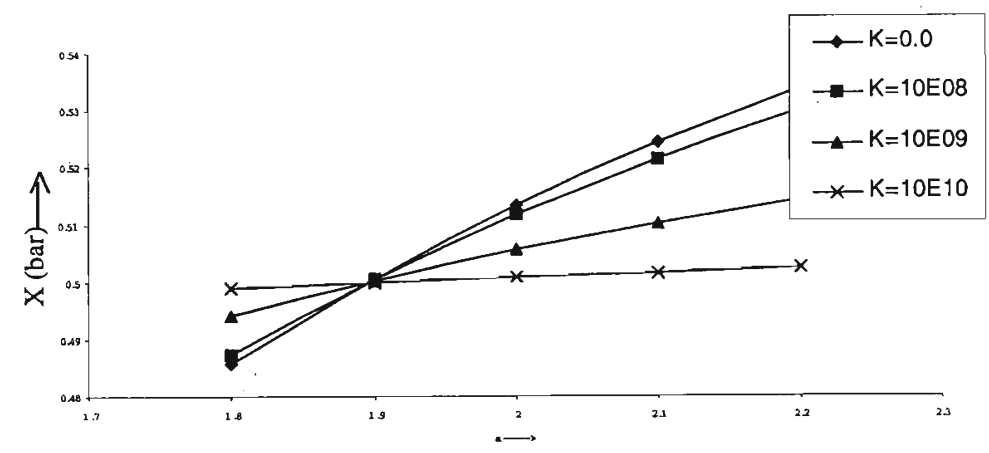

Figure 5: $\bar{X}$ vs $\mathrm{K}$ for various values of $\mathrm{a}$ for $\bar{\delta}=0.35, \bar{\beta}=2.77 \times 10^{-4}$

From Table $4 \overline{\mathrm{W}}$ increases with increasing values of a and decreases with increasing values of $\bar{\beta}$. 
Table 4: $\overline{\mathbf{W}}$ vs $\bar{\beta}$ for various values of a

\begin{tabular}{|c|c|c|c|c|c|c|}
\hline$\alpha^{2}$ & a & 1.8 & 1.9 & 2.0 & 2.1 & 2.2 \\
\hline 0.0 & & 0.4177950 & 0.4213546 & 0.4234699 & 0.4244518 & 0.4245434 \\
\hline $10^{-11}$ & & 0.4177691 & 0.4213279 & 0.4234425 & 0.4244245 & 0.4245164 \\
\hline $10^{-10}$ & & 0.4175330 & 0.4210870 & 0.4231989 & 0.4241796 & 0.4242709 \\
\hline $10^{-9}$ & & 0.4151714 & 0.4186782 & 0.4207618 & 0.4217286 & 0.4218176 \\
\hline
\end{tabular}

$\bar{\delta}=0.35, K=10^{9}, \bar{\beta}=2.77 \times 10^{7} \alpha^{2}$

From Table $5 \overline{\mathrm{F}}$ decreases when a increases. It decreases or increases when $\bar{\beta}$ increases accordingly as $\mathrm{a} \lesseqgtr \mathrm{a}_{2}, 1.9<\mathrm{a}_{2}<2.0$.

Table 5: $\overline{\mathbf{F}}$ vs $\bar{\beta}$ for various values of a

\begin{tabular}{|c|c|c|c|c|c|c|}
\hline$\overline{\alpha^{2}}$ & $\mathrm{a}$ & 1.8 & 1.9 & 2.0 & 2.1 & 2.2 \\
\hline 0.0 & & 0.9651934 & 0.9397183 & 0.9170077 & 0.8966164 & 0.8781847 \\
\hline $10^{-11}$ & & 0.9651908 & 0.9397179 & 0.9170097 & 0.8966198 & 0.8781895 \\
\hline $10^{-10}$ & & 0.9651700 & 0.9397151 & 0.9170235 & 0.8966495 & 0.8782341 \\
\hline $10^{-9}$ & & 0.9649586 & 0.9396868 & 0.9171667 & 0.8969527 & 0.878686 \\
\hline
\end{tabular}

$\bar{\delta}=0.35, \mathrm{~K}=10^{9}, \bar{\beta}=2.77 \times 10^{7} \alpha^{2}$

From Table $6 \overline{\mathrm{f}}$ decreases or increases accordingly as a or $\bar{\beta}$ increases.

Table 6: $\overline{\mathrm{f}}$ vs $\bar{\beta}$ for various values of a

\begin{tabular}{|c|c|c|c|c|c|c|}
\hline$\alpha^{2}$ & $\mathrm{a}$ & 1.8 & 1.9 & 2.0 & 2.1 & 2.2 \\
\hline 0.0 & & 2.3102081 & 2.2302313 & 2.1654613 & 2.1124103 & 2.0685394 \\
\hline $10^{-11}$ & & 2.3103454 & 2.2303717 & 2.1656063 & 2.1125543 & 2.0686824 \\
\hline $10^{-10}$ & & 2.3116016 & 2.2316411 & 2.1668856 & 2.1138439 & 2.0699842 \\
\hline $10^{-9}$ & & 2.3242412 & 2.2444131 & 2.1797764 & 2.1268480 & 2.0830965 \\
\hline
\end{tabular}

$\bar{\delta}=0.35, \mathrm{~K}=10^{9}, \bar{\beta}=2.77 \times 10^{7} \alpha^{2}$

From Table 7 the position of the centre of pressure shifts towards the outlet when a increases. However, it shifts towards the outlet or inlet accordingly as $\mathrm{a} \lesseqgtr \mathrm{a}_{2}, 1.8<\mathrm{a}_{2}<1.9$, when $\bar{\beta}$ increases 
Table 7: $\overline{\mathbf{X}}$ vs $\bar{\beta}$ for various values of a

\begin{tabular}{|c|c|c|c|c|c|c|}
\hline$\overline{\alpha^{2}}$ & $a$ & 1.8 & 1.9 & 2.0 & 2.1 & 2.2 \\
\hline 0.0 & & 0.4941046 & 0.5002806 & 0.5056180 & 0.5102714 & 0.5143570 \\
\hline $10^{-11}$ & & 0.4941047 & 0.5002802 & 0.5056174 & 0.5102704 & 0.5143555 \\
\hline $10^{-10}$ & & 0.4941058 & 0.5002772 & 0.5056108 & 0.5102605 & 0.5143430 \\
\hline $10^{-9}$ & & 0.4941176 & 0.5002466 & 0.5055439 & 0.5101622 & 0.5142168 \\
\hline
\end{tabular}

$\bar{\delta}=0.35, \mathrm{~K}=10^{9}, \bar{\beta}=2.77 \times 10^{7} \alpha^{2}$

\section{CONCLUSION}

The pressure and load capacity of the bearing can be increased considerably by increasing the strength of the external magnetic field. However, they are not much affected by the material parameter.

\section{Acknowledgement}

The authors are grateful to the referees for their useful suggestions and encouragement.

\section{References}

$1 \quad$ Abramovitz S. (1955). Journal of Franklin Institute 259 : 221.

2 Vinay Puri \& Patel C.M. (1983).The analysis of a pivoted porous slider bearing with a convex pad surface. Wear 88: 127.

3 Agrawal V.K. (1986). Magnetic fluid based porous inclined slider bearing. Wear 107: 133-139.

4 Paras Ram \& Verma P.D.S. (1999). Ferrofluid lubrication in porous inclined slider bearing. Indian Journal of Pure and Applied Mathematics 30(12) : 1273-1281.

5 Shah R.C. \& Bhat M.V. (2000). Squeeze film based on magnetic fluid in curved porous rotating circular plates. Journal of Magnetism and Magnetic Materials 208: 115-119.

6 Shah R.C. \& Bhat M.V. (2000). Squeeze film between two curved annular plates lubricated with magnetic fluid. Journal of Balkan Tribological Association 6: 184-189. 
7 Bhat M.V. (2003). Lubrication with a magnetic fluid. Team Sprit (India ) Pvt. Ltd. : 116. 